\title{
Discovering associative long-term synaptic modification and timing dependence of plasticity - a very brief and personal history
}

\author{
William B Levy ${ }^{1,2 *}$ \\ Department of Neurosurgery, University of Virginia, Charlottesville, VA, USA \\ 2 Department of Psychology, University of Virginia, Charlottesville, VA, USA \\ *Correspondence: wlevy@informedsimplifications.com
}

My long-term career goal was, and is, to construct credible quantitative theories of the biological basis of cognitive function. My approach is severely reductive which was a problem early on. We knew nothing about associative synaptic modification, the heart and soul of such cognitive theories. So motivated, I went to Virginia to work with my good friend Oswald Steward. In particular, I wanted to improve upon the observations of McNaughton (1977). His primary result was a demonstration of the intensity-dependence of long-term potentiation (LTP; a result that Bliss et al., 1973 looked for and somehow missed).

Before arriving in Virginia, I knew a twostimulating-electrode experiment (using provably independent and non-overlapping inputs) was necessary to produce an unequivocal demonstration of associative LTP. Unfortunately, I had no specific idea of how to do the experiment. When Ossie explained his discovery of the crossed entorhinal cortex to dentate gyrus pathway and showed me the baseline physiology ongoing in his lab, I seized on the opportunity presented by two independent monosynaptic pathways suitable for associative studies. A small number of layer II cells project monosynaptically to the ipsilateral and contralateral dentate gyrus. Using a one stimulating electrode in the left dentate gyrus and a second stimulating electrode in the right dentate gyrus yielded the necessary independent monosynaptic excitatory pathways converging on the same postsynaptic neurons. Although we began with just the intention of studying LTP, we quickly discovered a postsynapticdependent, synaptic long-term depression. This discovery was essentially unavoidable due to all the controls built into studying the bilateral path on both sides of the brain and studying the effect of brief high frequency stimulation of just one entorhinal cortex while recording from both the left and right dentate gyri. In essence, we were running two experiments at once, one by recording from the left dentate gyrus and one by recording from the right dentate gyrus. As we were finishing up the manuscript for this work (Levy and Steward, 1983), I began thinking about the next study, and these thoughts must have been influenced by my earlier training.

As background, there were my undergraduate studies in psychology and an awareness that stimuli used for conditioning worked best if they preceded a shock in negative reinforcement or a reactivated pressable lever for positive reinforcement. I also knew the Pavlovian paradigms (see discussion in Levy and Steward, 1983) and was aware of associative timing experiments in this behavioral context.

Once again, by recording on both sides of the brain while performing an associative timing experiment, we automatically examined a pre-then-post as well as a post-then-pre activity paradigm. That is, if the left stimulating electrode was active before the right stimulating electrode, then recording from the left dentate gyrus was a depression paradigm while recording from the right dentate gyrus was a potentiation paradigm. Thus the spike-timing effect was discovered. The published experiments themselves were completed before the end of 1980 and were reported at a wonderful little gathering in 1981 at Brown University (hosted by Leon Cooper and eventually published in 1985; Levy, 1985).

Shortly after our publication, in which postsynaptic cell firing in the dentate gyrus was controlled by each ipsilateral pathway, Gustafsson et al. (1987) published a timing study in CA1. They used intracellular control of postsynaptic excitation. However, their temporal resolution was lower than our work. With later studies came higher temporal resolution (Bi and Poo, 1998).
Our later work showed that the accuracy of timing was better than $20 \mathrm{~ms}$ and - by using large, multiple TTX injections - that we really were in complete control of the EC inputs (Lopez et al., 1990). Another pretty set of studies by Ossie's student Geoff White show that STDP could be localized within a dendritic region (White et al., 1988, 1990). Later in the decade, Holmes and Levy (1990) worked out the biophysics of the potentiation portion of the spike timing rule.

My major experimental regret is the drug experiments that I shunned early on (but see Desmond et al., 1991). My undergraduate and graduate experience with neuropharmacology left me with skepticism concerning drug specificity, and I was mistaken not to accept and use the rapidly developing glutamate receptor pharmacology of the early 1980s. Nevertheless, the two original Levy and Steward $(1979,1983)$ studies were enough to embark on my goal of quantitative modeling with half of the STDP playing a major role in later theoretical work (Levy and Desmond, 1985; Levy, 1989; Levy et al., 1990).

\section{REFERENCES}

Bi, G. Q., and Poo, M. M. (1998). Synaptic modification in cultured hippocampal neurons: dependence on spike timing, synaptic strength, and postsynaptic cell type. J. Neurosci. 18, 10464-10472.

Bliss, T. V.P., and Lømo, T. (1973). Long-lasting potentiation of synaptic transmission in the dentate area of the anaesthetized rabbit following stimulation of the perforant path. J. Physiol. 232, 331-356.

Desmond, N. L., Colbert, C. M., Zhang, D. X., and Levy, W. B. (1991). NMDA receptor antagonists block the induction of long-term depression in the hippocampal dentate gyrus of the anesthetized rat. Brain Res. 552, 93-98.

Gustafsson, B., Wigström, H., Abraham, W. C., and Huang, Y.-Y. (1987). Long-term potentiation in the hippocampus using depolarizing current pulses as the conditioning stimulus to single volley synaptic potentials. J. Neurosci. 7, 774-780.

Holmes, W. R., and Levy, W. B. (1990). Insights into associative long-term potentiation from computational 
models of NMDA receptor-mediated calcium influx and intracellular calcium concentration changes. $J$. Neurophysiol. 63, 1148-1168.

Levy, W. B. (1985). "Associative changes at the synapse: LTP in the hippocampus," in Synaptic Modification, Neuron Selectivity and Nervous System Organization, eds W. B Levy, J. Anderson, and S. Lehmkuhle (Hillsdale, NJ: Lawrence Erlbaum Assoc., Inc.), 5-33.

Levy, W. B. (1989). "A computational approach to hippocampal function," in Computational Models of Learning in Simple Neural Systems, eds R. D. Hawkins and G. H. Bower (New York: Academic Press), 243-305.

Levy, W. B., Colbert, C. M., and Desmond, N. L. (1990). "Elemental adaptive processes of neurons and synapses: a statistical/computational perspective," in: Neuroscience and Connectionist Models, eds M. A. Gluck and D. E. Rumelhart (Hillsdale, NJ: Lawrence Erlbaum Assoc., Inc.), 187-235.

Levy, W. B., and Desmond, N. L. (1985). “The rules of elemental synaptic plasticity," in Synaptic Modification,
Neuron Selectivity and Nervous System Organization, eds W. B Levy, J. Anderson, and S. Lehmkuhle (Hillsdale, NJ: Lawrence Erlbaum Assoc., Inc.), 105-121.

Levy, W. B., and Steward, O. (1979). Synapses as associative memory elements in the hippocampal formation. Brain Res. 175, 233-245.

Levy, W. B., and Steward, O. (1983). Temporal contiguity requirements for long-term associative potentiation/depression in the hippocampus. Neuroscience 8, 791-797.

Lopez, H. S., Burger, B., Dickstein, R., Desmond, N. L., and Levy, W. B. (1990). Associative synaptic potentiation and depression: quantification of dissociable modifications in the hippocampal dentate gyrus favors a particular class of synaptic modification equations. Synapse 5, 33-47.

McNaughton, B. L. (1977). Dissociation of short-and long-lasting modification of synaptic efficacy at the terminals of the perforant path. Seventh Annual Meeting of the Society for Neuroscience, Anaheim, CA, Abstract.
White, G., Levy, W. B., and Steward, O. (1988). Evidence that associative interactions between synapses during the induction of long-term potentiation occur within local dendritic domains. Proc. Natl. Acad. Sci. U.S.A. $85,2368-2372$.

White, G., Levy, W. B., and Steward, O. (1990). Spatial overlap between populations of synapses determines the extent of their associative interaction during the induction of long term potentiation and depression. J. Neurophysiol. 64, 1186-1198.

Received: 07 September 2010; accepted: 05 November 2010; published online: 02 December 2010.

Citation: Levy WB (2010) Discovering associative longterm synaptic modification and timing dependence of plasticity - a very brief and personal history. Front. Syn. Neurosci. 2:149. doi: 10.3389/fnsyn.2010.00149

Copyright (C) 2010 Levy. This is an open-access article subject to an exclusive license agreement between the authors and the Frontiers Research Foundation, which permits unrestricted use, distribution, and reproduction in any medium, provided the original authors and source are credited. 